\title{
DEFINABLE SINGULARITY
}

\author{
WILLIAM J. MITCHELL
}

\begin{abstract}
The main result of this paper is a characterization of singular cardinals in terms of the core model, assuming that there is no model of $\exists \kappa o(\kappa)=$ $\kappa^{++}$. This characterization is used to prove a result in infinitary Ramsey theory. In the course of the proof we develop a simplified statement of the covering lemma for sequences of measures which avoids the use of mice. We believe that this development will be capable of isolating almost all applications of the covering lemma from the detailed structure of the core model.
\end{abstract}

\section{INTRODUCTION}

This paper contains results on three different topics. The first, Theorem 1.2, is a counterexample to a question in infinitary Ramsey theory, generalizing a result in [Mi89b]. The second topic, which was first studied because it was required for Theorem 1.2 but which became the main result of this paper, is a generalization to sequences of measures of the result of Dodd and Jensen [D] that if $0^{\dagger}$ does not exist and $\kappa$ is a singular cardinal which is not singular in $K$ or in $L(\mu)$, then there is a Prikry sequence cofinal in $\kappa$ which is definable up to initial segments. We show that if there is no model of $\exists \kappa o(\kappa)=\kappa^{++}$and $\kappa$ is any singular cardinal, then there is a cofinal subset of $\kappa$ witnessing the singularity of $\kappa$ which is (in an appropriate sense) definable. The third topic, which was originally inspired by frustration with attempts at a reasonable exposition of the second topic, is a statement of the covering lemma for sequences of measures which avoids the mention of mice. This considerably simplifies the statement and at least some applications (but not the proof) of the covering lemma.

The rest of the introduction will discuss three topics in more detail. The topics will be given in chronological order as in the last paragraph.

1.1. Definition. We write $[X]^{\omega}$ for $\{c \subset X$ : ordertype $(c)=\omega\}$ and $\Re_{f}$ for the set of equivalence classes of reals modulo "equality except on an initial segment." The expression $\kappa \stackrel{\mathrm{OD}}{\rightarrow}(\omega)_{\Re_{f}}^{\omega}$ means that for every ordinal definable function $f:[\kappa]^{\omega} \rightarrow \Re_{f}$ there is $c \in[\kappa]^{\omega}$ such that $f$ is constant on $[c]^{\omega}$.

Received by the editors September 6, 1989 and, in revised form, February 5, 1990.

1980 Mathematics Subject Classification (1985 Revision). Primary 03E47; Secondary 03E35, $03 \mathrm{E} 45,03 \mathrm{E} 55$.

Key words and phrases. Singular cardinals, core model, covering lemma.

The author's research was partly supported by grant \#OMS-8164447 from the National Science Foundation. 
1.2. Theorem. Suppose that $\kappa \stackrel{\mathrm{OD}}{\rightarrow}(\omega)_{\Re_{f}}^{\omega}$ but $\left(2^{\omega}\right)^{+} \underset{\nrightarrow}{\mathrm{OD}}(\omega)_{\Re_{f}}^{\omega}$. Then there is an inner model having a cardinal $\alpha$ such that $o(\alpha)$ is measurable. Furthermore, if $\kappa \stackrel{\mathrm{OD}}{\rightarrow}(\omega)_{\Re_{f}}^{\omega}$ holds and there is a function $f:\left[\left(2^{\omega}\right)^{+}\right]^{\omega} \rightarrow \Re_{f}$ serving as a counterexample to $\left(2^{\omega}\right)^{+} \stackrel{\mathrm{OD}}{\rightarrow}(\omega)_{\Re_{f}}^{\omega}$ such that $f(c)=f\left(c^{\prime}\right)$ whenever $c$ and $c^{\prime}$ are countable sequences such that $c \backslash \delta=c^{\prime} \backslash \delta$ for some $\delta<\sup (c)$, then there is an inner model of $o(\kappa)=\kappa^{++}$.

The proof of this theorem is given in $\S 4$. It requires finding, for each singular cardinal $\kappa$, a cofinal subset $C$ of $\kappa$ which witnesses the singularity of $\kappa$ and is nearly enough definable. The theorem was proved in [Mi89b] with the conclusion weakened to assert only the existence of more than a measurable cardinal. That proof used the Dodd-Jensen covering lemma [D], which asserts that if $\kappa$ is a singular cardinal and $0^{\dagger}$ does not exist, then one of three possibilities must hold: (1) there is no model $L(\mu)$ with a measurable cardinal, and $\kappa$ is singular in the core model $K,(2)$ there is a model $L(\mu)$ and $\kappa$ is singular there, or (3) there is a model $L(\mu)$, where $\mu$ is a measure on $\kappa$ and $\kappa$ is singular because there is a set $C$ which is Prikry generic over $L(\mu)$. In cases (1) and (2) a definable witness to the singularity of $\kappa$ is given by the definable well-ordering of $K$ and $L(\mu)$. In the last case, which is the most interesting, the set $C$ is not definable but it does have the following maximality property: if $C^{\prime}$ is any other Prikry sequence, then $C^{\prime} \backslash C$ is finite. Since any other maximal Prikry sequence must be eventually equal to $C$, the sequence $C$ is definable up to an initial segment.

This led to the problem of finding, under the weaker assumption that there is no model of $\exists \kappa o(\kappa)=\kappa^{++}$, a witness to the singularity of a singular cardinal which is definable (in some appropriate sense), and hence to the characterization of singular cardinals which is the second phase of the research and which we regard as the main result of this paper. This characterization is outlined in Table 1 and proved in $\S 3$. It turns out not to be true that every singular cardinal has a witness which is definable up to an initial segment (case 4 is the exception) but we do obtain a maximality property, and hence a definability property, which seems reasonable and is strong enough to prove Theorem 1.2.

The table characterizes the singular cardinals $\kappa$ such that $\lambda^{\omega}<\kappa$ for all $\lambda<\kappa$, under the assumption that there is no inner model of $\exists \kappa o(\kappa)=\kappa^{++}$. The ordinal $\beta$ used in the definition of the cases is the least ordinal such that $\mathscr{F}(\kappa, \beta)$ is not generated by indiscernibles, or $o(\kappa)$ if there is no such ordinal. The precise definition of $\beta$ and of other terms used in the table appears below and in $\S 2$. The proof of the assertions in this table is given in $\S 3$. In order to save space in the table we have written $\mathbf{K}$ for $K(\mathscr{F})$, the maximal core model for sequences of measures.

The following paragraphs define most of the terms in the table. Throughout this paper $o(\alpha)$ means $o^{K(\mathscr{F})}(\alpha)$. We use boldface $\mathbf{c}$ for the coherence function for $K(\mathscr{F})$ : that is, $\mathbf{c}\left(\kappa, \lambda^{\prime}, \lambda\right)$ is the least function $g \in{ }^{\kappa} \kappa \cap K(\mathscr{F})$ in the ordering of $K(\mathscr{F})$ such that $\lambda^{\prime}=[g]_{\mathscr{F}(\kappa, \lambda)}$. 
TABLE 1

\begin{tabular}{|c|c|c|c|c|}
\hline case & $\begin{array}{c}\text { description } \\
\text { of case }\end{array}$ & $\operatorname{cf}(\kappa)$ & $\begin{array}{c}\text { description of } \\
\text { cofinal sequence }\end{array}$ & $\begin{array}{c}\text { typical } \\
\text { forcing }\end{array}$ \\
\hline 1 & $\operatorname{cf}^{\mathbf{K}}(\kappa)<\kappa$ & $\operatorname{cf}\left(\operatorname{cf}^{\mathbf{K}}(\kappa)\right)$ & taken from $\mathbf{K}$ & \\
\hline 2 & $\beta=\gamma+1$ & $\omega$ & $\begin{array}{c}\text { Maximal } \\
\text { Prikry sequence } \\
\text { for } \mathscr{F}(\kappa, \gamma)\end{array}$ & Prikry forcing \\
\hline 3 & $\operatorname{cf}(\beta)>\kappa$ & $\omega$ & $\begin{array}{c}\text { Maximal accumulation } \\
\text { sequence for } \beta\end{array}$ & Gitik [Gi] \\
\hline 4 & $\operatorname{cf}^{\mathbf{K}}(\beta)=\kappa$ & $\omega$ & $\begin{array}{c}c_{0}=s^{C}(0, \nu) \\
c_{i+1}=s^{C}\left(f\left(c_{i}\right), \nu\right)\end{array}$ & $\begin{array}{c}\text { Radin forcing, } \\
o(\kappa)=\kappa\end{array}$ \\
\hline 5 & $\operatorname{cf}^{\mathbf{K}}(\beta)<\kappa$ & ${\operatorname{cf}\left(\mathrm{cf}^{\mathbf{K}}(\beta)\right)}^{c_{l}=s^{C}(f(l), \nu)}$ & $\begin{array}{c}\text { Magidor forcing, } \\
o(\kappa)<\kappa\end{array}$ \\
\hline
\end{tabular}

1.3. Definition. (1) A generating sequence for a measure $\mathscr{F}(\kappa, \lambda)$ is a cofinal subset $C$ of $\kappa$ having a function $g$ such that $g(\nu) \leq o(\nu)$ for all sufficiently large $\nu \in C$ and such that any set $x \in K(\mathscr{F})$ is in $\mathscr{F}(\kappa, \lambda)$ if and only if for every sufficiently large $\nu \in C$ we have

$$
\begin{array}{cl}
\nu \in X & \text { if } g(\nu)=o(\nu), \\
x \cap \nu \in \mathscr{F}(\nu, g(\nu)) & \text { if } g(\nu)<o(\nu) .
\end{array}
$$

(2) $\beta$ is the least ordinal less than $o(\kappa)$ such that there is no generating sequence for $\mathscr{F}(\kappa, \beta)$, or $\beta=o(\kappa)$ if there is no such ordinal.

The simplest example of a generating sequence for $\mathscr{F}(\kappa, \lambda)$ is a Prikry sequence $C$ for $\mathscr{F}(\kappa, \lambda)$, with $g(\nu)=o(\nu)$ for all $\nu \in C$. The same sequence $C$ is also a generating sequence for every measure $\mathscr{F}\left(\kappa, \lambda^{\prime}\right)$ with $\lambda^{\prime}<\lambda$, using the coherence function $g=\mathbf{c}\left(\kappa, \lambda^{\prime}, \lambda\right)$. If $o(\kappa)=\omega_{1}$ then it is possible, using Magidor forcing [Ma], to add a cofinal sequence $C$ consisting of one indiscernible for each of the measures $\mathscr{F}(\kappa, \lambda)$ with $\lambda<\omega_{1}$. Then this sequence is a generating sequence for $\mathscr{F}(\kappa, \lambda)$ for each $\lambda<\omega_{1}$, using the constant function $g(\nu)=\lambda$, since $\mathbf{c}\left(\kappa, \lambda, \lambda^{\prime}\right)(\nu)=\lambda$ for all $\lambda^{\prime}<o(\kappa)$ and all $\lambda<\min \left(\lambda^{\prime}, \kappa\right)$.

The set $C$ referred to in cases 4 and 5 is a set of indiscernibles which contains generating sequences for every measure below $\mathscr{F}(\kappa, \beta)$ and which is maximal in a sense to be described in $\S 2$. The function $f$ is any continuous function in $K(\mathscr{F})$ which maps $\operatorname{cf}^{K(\mathscr{F})}(\beta)$ cofinally into $\beta$ and $\nu$ is any sufficiently large ordinal below $\kappa$. The precise definition of the function $s^{C}$ will be given in $\S 2$, but the idea is that whenever $\lambda<\beta$ and $\nu<\kappa$ then $s^{C}(\lambda, \nu)$ is the smallest member of $C \backslash(\nu+1)$ which is an indiscernible for some measure $\mathscr{F}\left(\kappa, \lambda^{\prime}\right)$ with $\lambda^{\prime} \geq \lambda$. If $\lambda<\kappa$ then this implies that $s^{C}(\lambda, \nu)$ is the smallest member $\gamma$ of $C \backslash(\nu+1)$ such that $o(\gamma) \geq \lambda$. 
1.4. Definition. An accumulation sequence for an ordinal $\lambda \leq o(\kappa)$ is a cofinal set $A=\left\{\alpha_{n}: n \in \omega\right\} \in[\kappa]^{\omega}$ such that $A$ is not a generating sequence for $\mathscr{F}(\kappa, \lambda)$, and such that for any sequence $\vec{\delta}$ such that $\delta_{n}<\alpha_{n}$ for all $n \in \omega$ and any measure $\mathscr{F}\left(\kappa, \lambda^{\prime}\right)$ with $\lambda^{\prime}<\lambda$ there is a generating sequence $C$ for $\mathscr{F}\left(\kappa, \lambda^{\prime}\right)$ such that $C \cap\left(\alpha_{n} \backslash \delta_{n}\right) \neq \varnothing$ for all $n \in \omega$.

A sequence $A$ of accumulation points for $\lambda$ is maximal if any other sequence of accumulation points for $\lambda$ is contained in $A$ except for an initial set.

$\S 2$ below contains the simplified covering lemma which is used in proving the assertions in Table 1. The covering lemma is given as two lemmas, Lemmas 2.5 and 2.15. The separation into two lemmas is for ease of exposition and they are best regarded together as "the covering lemma." The proof of these lemmas requires results from [Mi84 and Mi89a] and an understanding of the apparatus of mice used to define the core model $K(\mathscr{F})$ and to prove the covering lemma as given in those papers. The rest of the paper makes no such assumption, and it is intended that all of this paper except the proofs of Lemmas 2.5 and 2.15 should be accessible to any reader who has been exposed to coherent sequences of measures as in [Mi74], to Prikry, Magidor, and Radin forcing, and to some background in applications of the Dodd-Jensen core model theory. We will be using the following facts about $K(\mathscr{F})$ : The core model $\mathscr{F}$ is a coherent sequence of measures which is similar to (and in fact may be equal to) $L(\mathscr{F})$. Unlike general models $L(\mathscr{U})$, the sequence $\mathscr{F}$ and the model $K(\mathscr{F})$ are unique and definable without parameters by a $\Pi_{2}$ formula. In addition to Lemmas 2.5 and 2.15 we use the basic consequence of the covering lemma, that $\left(\kappa^{+}\right)^{K(\mathscr{F})}=\kappa^{+}$whenever $\kappa$ is a singular cardinal.

The last section of this paper, $\S 5$, contains some notes on generalizations of results in this paper and some open questions.

\section{INDISCERNIBLES AND THE COVERING LEMMA}

Roughly speaking, a set of indiscernibles is a generating sequence $C$ such that each member $\nu \in C$ can be assigned as an indiscernible for some particular measure. Two key ideas in this paper are clauses (2) and (3) of Proposition 2.2, which state that if such an assignment exists then it is essentially unique and a member of $K(\mathscr{F})$, and Lemmas 2.5 and 2.15, which state the covering lemma in terms of such assignments. In particular, Lemma 2.5 implies that every generating sequence $C$ can be given such an assignment, provided that $|C|^{\omega}<$ $\kappa$.

2.1. Definition. If $C \subset \kappa$ then an assignment for $C$ is a function $\beta: C \rightarrow$ $o(\kappa)$ such that

$$
\forall h \in K(\mathscr{F}) \exists \delta<\kappa \forall \nu \in C \backslash \delta \forall x \in h^{“} \nu \quad \nu \in x \Leftrightarrow x \in \mathscr{F}(\kappa, \beta(\nu)) .
$$

A set of indiscernibles in $\kappa$ is a set $C \subset \kappa$ such that $C$ has an assignment and $|C|^{\omega}<|\kappa|$. 
Since any assignment for a terminal segment $C \backslash \delta$ of $C$ can be extended trivially to a assignment for all of $C$ (for example, by setting $\beta(\nu)=0$ for $\nu \in C \cap \delta$ ), we will call $\beta$ an assignment for $C$ if it is an assignment for a terminal segment of $C$.

Recall that the assumption in clause $(2)$ that $\left(\kappa^{+}\right)^{K(\mathscr{F})}=\kappa^{+}$is a basic consequence of the covering lemma, provided that there is no model of $\exists \kappa o(\kappa)=$ $\kappa^{++}$.

2.2. Proposition. (1) If $\beta$ is an assignment for $C \supset C^{\prime}$ then $\beta\left\lceil C^{\prime}\right.$ is an assignment for $C^{\prime}$.

(2) If $\beta$ is an assignment for $C$ and $\left(\kappa^{+}\right)^{K(\mathscr{F})}=\kappa^{+}$then there are functions $h$ and $g$ in $K(\mathscr{F})$ such that for some $\delta<\kappa$ and all $\nu \in C \backslash \delta$ we have $\beta(\nu)=g(\nu) \in h^{\prime \prime} \nu$.

(3) If $\beta$ and $\beta^{\prime}$ are each assignments for $C$ and $\left(\kappa^{+}\right)^{K(\mathscr{F})}=\kappa^{+}$then there is $\delta<\kappa$ such that $\beta \uparrow(C \backslash \delta)=\beta^{\prime} \uparrow(C \backslash \delta)$.

Proof. Clause (1) is immediate. For (2), note that, since $o(\kappa)<\left(\kappa^{++}\right)^{K(\mathscr{F})}$, the hypothesis implies that there is a one-to-one function $h: \kappa \rightarrow \kappa^{++}$in $K(\mathscr{F})$ such that range $(\beta) \subset \operatorname{range}(h)$. Let $\left(x_{\nu}: \nu<\kappa\right) \in K(\mathscr{F})$ be a disjoint sequence of subsets of $\kappa$ such that $x_{\nu} \in \mathscr{F}(\kappa, h(\nu))$ and let $z=\left\{\nu: \exists \nu^{\prime}<\nu \nu \in x_{\nu^{\prime}}\right\}$. Then $z \in \mathscr{F}(\kappa, h(\nu))$ for all $\nu<\kappa$, so $C$ is eventually contained in $z$. For $\nu \in z$ define $g(\nu)$ to be $h\left(\nu^{\prime}\right)$, where $\nu^{\prime}<\nu$ is the unique ordinal such that $\nu \in x_{\nu^{\prime}}$. Then every sufficiently large $\nu \in C$ satisfies the statements $\nu \in z$ and $\forall \xi<\nu\left(\nu \in x_{\xi} \Leftrightarrow x_{\xi} \in \mathscr{F}(\kappa, \beta(\nu))\right)$, which together imply $\beta(\nu)=g(\nu)$.

The function $g$ obtained in the last paragraph depended only on the function $h$. If $\beta$ and $\beta^{\prime}$ are two assignments for $C$ then a function $h$ can be chosen so that range $(h)$ contains both $\operatorname{range}(\beta)$ and $\operatorname{range}\left(\beta^{\prime}\right)$. It follows that $\beta$ and $\beta^{\prime}$ are both eventually equal to $g$ and hence are eventually equal. This completes the proof of clause (3) and of the proposition.

Thus we can pick, for each set $C$ of indiscernibles, a function $\beta^{C} \in K(\mathscr{F})$ such that $\beta^{C}{ }_{i C}$ is an assignment for $C$. The function $\beta^{C}{ }_{i}$ is unique up to initial segments, and $\beta^{C}$ is unique up to sets which are of measure 0 in $\mathscr{F}\left(\kappa, \beta^{C}(\nu)\right)$ for every sufficiently large $\nu \in C$.

If $\eta<o(\kappa)$ and $\xi<\kappa$ then we write $s^{C}(\eta, \xi)$ for the least member $\nu$ of $C$ above $\xi$, if there is one, such that $\beta^{C}(\nu) \geq \eta$. For convenience we will say that $s^{C}(\eta, \xi)=\kappa$ if there is no such member $\nu$ of $C$. It should be noted that this definition is slightly different from that in [Mi89a]. The ordinal $s^{C}(\kappa, \eta, \xi)$ as defined in that paper is equal to $\nu=s^{C}(\eta, \xi)$ as defined in this paper if $\beta^{C}(\nu)=\eta$, and is undefined otherwise.

2.3. Proposition. Suppose $C$ is a set of indiscernibles and $f$ is a function in $K(\mathscr{F})$ such that $f(\nu)<o(\nu)$ for all $\nu \in C$. Then there is a sequence $\left(\xi_{\alpha}: \alpha<\kappa\right)$ in $K(\mathscr{F})$ such that for every sufficiently large member $\nu$ of $C$ there is $\alpha(\nu)<\nu$ such that $f(\nu)=\mathbf{c}\left(\kappa, \xi_{\alpha(\nu)}, \beta^{C}(\nu)\right)(\nu)$. Furthermore, if 
$x \subset \kappa$ is in $K(\mathscr{F})$ then there is $\delta<\kappa$ such that for all $\nu \in C \backslash \delta$ we have $x \cap \nu \in \mathscr{F}(\kappa, f(\nu)) \Leftrightarrow x \in \mathscr{F}\left(\kappa, \xi_{\nu(\alpha)}\right)$.

Proof. We first observe that

$$
\left\{\eta: \beta^{C}(\eta)=\beta^{C}(\nu)\right\} \in \mathscr{F}\left(\kappa, \beta^{C}(\nu)\right)
$$

for all sufficiently large $\nu \in C$, since (using the notation of the proof of Proposition 2.2) if $\xi<\nu$ is the ordinal such that $\beta^{C}(\nu)=h(\xi)$ then $\left\{\eta: \beta^{C}(\eta)=\right.$ $\left.\beta^{C}(\nu)\right\}=x_{\xi} \in \mathscr{F}(\kappa, h(\xi))=\mathscr{F}\left(\kappa, \beta^{C}(\nu)\right)$.

For $\nu<\kappa$ set $\xi_{\nu}$ equal to $[f]_{\mathscr{F}_{\left(\kappa, \beta^{C}(\nu)\right)}}$ and define $\alpha(\eta)$ to be the least $\alpha<\eta$, if there is one, such that $f(\eta)=\mathbf{c}\left(\kappa, \xi_{\alpha}, \beta^{C}(\eta)\right)(\eta)$. Then the functions $\xi$ and $\alpha$ are both in $K(\mathscr{F})$. Since $\left\{\eta: f(\eta)=\mathbf{c}\left(\kappa, \xi_{\nu}, \beta^{C}(\nu)\right)(\eta)\right\} \in$ $\mathscr{F}\left(\kappa, \beta^{C}(\nu)\right)$, equation (1) implies

$$
\{\eta: \alpha(\eta) \text { exists and } \alpha(\eta) \leq \nu<\eta\} \in \mathscr{F}\left(\kappa, \beta^{C}(\nu)\right) .
$$

Since $\beta^{C}$ is an assignment, it follows that $\alpha(\nu)$ exists and is smaller than $\nu$ for all sufficiently large $\nu \in C$.

Now suppose that $x \in K(\mathscr{F})$. Then $x \in \mathscr{F}\left(\kappa, \xi_{\alpha(\nu)}\right)$ if and only if

$$
\left\{\eta: x \cap \eta \in \mathscr{F}\left(\eta, \mathbf{c}\left(\kappa, \xi_{\alpha(\nu)}, \beta^{C}(\nu)\right)(\eta)\right)\right\} \in \mathscr{F}\left(\kappa, \beta^{C}(\nu) .\right.
$$

Now since $\{\eta: \alpha(\eta)<\eta\} \in \mathscr{F}\left(\kappa, \beta^{C}(\nu)\right), \alpha(\eta)$ is constant on a set in $\mathscr{F}\left(\kappa, \beta^{C}(\nu)\right)$, and it follows that for sufficiently large $\nu \in C$ we have $\alpha(\eta)=$ $\alpha(\nu)$ on a set in $\mathscr{F}\left(\kappa, \beta^{C}(\nu)\right)$. Then by (1) we have $\mathrm{c}\left(\kappa, \xi_{\alpha(\nu)}, \beta^{C}(\nu)\right)(\eta)=$ $\mathbf{c}\left(\kappa, \xi_{\alpha(\eta)}, \beta^{C}(\eta)\right)(\eta)=f(\eta)$ on a set in $\mathscr{F}\left(\kappa, \beta^{C}(\nu)\right)$, so that equation (3) is equivalent to $\{\eta: x \cap \eta \in \mathscr{F}(\eta, f(\eta))\} \in \mathscr{F}\left(\kappa, \beta^{C}(\nu)\right)$, which for sufficiently large $\nu \in C$ is equivalent to $x \cap \nu \in \mathscr{F}(\kappa, f(\nu))$.

2.4. Corollary. If $C$ is a set of indiscernibles and $\lambda<o(\kappa)$ then $C$ is a generating sequence for $\mathscr{F}(\kappa, \lambda)$ if and only if $\beta^{C}(\nu) \geq \lambda$ for all sufficiently large $\nu \in C$. In this case the unique function $g$ (up to initial segment) witnessing that $C$ is a generating sequence for $\mathscr{F}(\kappa, \lambda)$ is given by

$$
g(\nu)= \begin{cases}o(\nu) & \text { if } \beta^{C}(\nu)=\lambda, \\ \mathbf{c}\left(\kappa, \lambda, \beta^{C}(\nu)\right)(\nu) & \text { if } \beta^{C}(\nu)>\lambda .\end{cases}
$$

Proof. It is easy to see that if $\beta^{C}(\nu) \geq \lambda$ for all sufficiently large $\nu \in C$ then $C$ does generate $\mathscr{F}(\kappa, \lambda)$ via the indicated function $g$. Now suppose that $C$ generates $\mathscr{F}(\kappa, \lambda)$ via a function $f \in K(\mathscr{F})$. Consider first $C_{0}=\{\nu \in$ $C: f(\nu)=o(\nu)\}$. There is a set $x \in K(\mathscr{F})$ such that $x \in \mathscr{F}(\kappa, \lambda)$ but $x \notin$ $\mathscr{F}\left(\kappa, \beta^{C}(\nu)\right)$ for any $\nu<\kappa$ such that $\beta^{C}(\nu) \neq \lambda$. Then by the assumption on $f, C_{0}$ is eventually contained in $x$ and it immediately follows that for all sufficiently large $\nu \in C_{0}$ we have $\beta^{C}(\nu)=\lambda$ and hence $f(\nu)=o(\nu)=g(\nu)$.

Now consider $C_{1}=\{\nu \in C: f(\nu)<o(\nu)\}$. Let the function $\left(\xi_{\alpha}: \alpha<\kappa\right)$ be as given by the last proposition and let $x$ be a set in $K(\mathscr{F})$ such that 
$x \in \mathscr{F}(\kappa, \lambda)$ and $x \notin \mathscr{F}\left(\kappa, \xi_{\alpha}\right)$ whenever $\xi_{\alpha} \neq \lambda$. Then since $C$ generates $\mathscr{F}(\kappa, \lambda)$ via the function $f, x \cap \nu \in \mathscr{F}(\nu, f(\nu))$ for all sufficiently large $\nu \in C_{1}$. By Proposition 2.1, for all sufficiently large $\nu \in C_{1}$ there is $\alpha(\nu)<\nu$ such that $f(\nu)=\mathbf{c}\left(\kappa, \xi_{\alpha(\nu)}, \beta^{C}(\nu)\right)(\nu)$ and $x \cap \nu \in \mathscr{F}(\nu, f(\nu))$ implies that $x \in \mathscr{F}\left(\kappa, \xi_{\alpha(\nu)}\right)$. It follows from the choice of $x$ that $\xi_{\alpha(\nu)}=\lambda$ and hence $f(\nu)=\mathbf{c}\left(\kappa, \lambda, \beta^{C}(\nu)\right)(\nu)=g(\nu)$.

Thus the function $f$ is equal to $g$ on all but a bounded subset of $C$. It follows immediately that $\lambda \leq \beta^{C}(\nu)$ for all but a bounded subset of $C$.

The next lemma is the first installment of the covering lemma.

2.5. Lemma. Suppose that $D \subset \kappa$ and $|D|^{\omega}<|\kappa|$. Then there is a set $C$ of indiscernibles such that:

(1) There is a function $h \in K(\mathscr{F})$ such that $h^{\prime \prime} \nu \cap(\kappa \backslash \nu) \neq \varnothing$ for every member $\nu$ of $D \backslash C$.

(2) $C$ contains all its limit points of cofinality $\omega$.

(3) If $\nu$ is any limit point of $C$ then $C \cap \nu$ is a set of indiscernibles in $\nu$.

(4) If $\nu$ is any limit point of $C$ in $C$ then there is $\xi \in C \cap \nu$ such that $\nu=s^{C}\left(\beta^{C}(\nu), \xi\right)$.

(5) For any other set $C^{\prime}$ of indiscernibles there is a $\delta<\kappa$ such that $s^{C}(\eta, \nu) \leq s^{C^{\prime}}(\eta, \nu)$ for every $\nu \in C \backslash \delta$ and any $\eta \in \operatorname{range} \beta^{C}$.

Proof. We assume that the reader is familiar with [Mi89a]. Pick a set $X \prec H_{\kappa^{++}}$ as in that paper with ${ }^{\omega} X \subset X$ and $D \subset X$. Let $\mathbf{m}$ be the mouse, let $\mathscr{C}^{\mathbf{m}}$ be the system of indiscernibles which covers $X$, and let $h^{\mathbf{m}}$ be the canonical skolem function for $\mathbf{m}$. The desired set $C$ of indiscernibles will be $\bigcup\left\{\mathscr{C}^{\mathbf{m}}(\kappa, \lambda): \lambda<\right.$ $\left.o^{\mathbf{m}}(\kappa)\right\}$ (or more properly, a terminal subset of this union).

Clauses (1) and (2) are immediate; clause (2) because ${ }^{\omega} X \subset X$ and clause (1) because every ordinal $\nu \in D \backslash C$ either is not an indiscernible in $\mathscr{C}^{\mathbf{m}}$, and hence is in $h^{\mathrm{m} \text { " }} \nu$, or else is in $\mathscr{C}^{\mathrm{m}}(\alpha, \lambda)$ for some $\alpha$ such that $\nu<\alpha<\kappa$ and some $\lambda<o(\alpha)$, so that $\alpha \in h^{\mathrm{m}} " \nu \cap(\kappa \backslash \nu)$.

Now we show that $C$ is a set of indiscernibles. First we use $\mathscr{C}^{m}$ to obtain an assignment for $C$ : Let $\beta^{\mathbf{m}} \leq o^{\mathbf{m}}(\kappa)$ be the least ordinal $\eta$ such that $\bigcup_{\lambda \geq \eta} \mathscr{C}^{\mathbf{m}}(\kappa, \lambda)$ is bounded in $\kappa$. Define $\tau^{\mathbf{m}}\left(\beta^{\mathbf{m}}\right)=\beta$, the least ordinal less than or equal to $o(\kappa)$ such that either $\beta=o(\kappa)$ or $\mathscr{F}(\kappa, \beta)$ is not generated by any set of indiscernibles, and for $\eta<\beta^{\mathrm{m}}$ define $\tau^{\mathrm{m}}(\eta)$ to be the unique ordinal $\lambda<o(\kappa)$ such that $\mathscr{F}(\kappa, \lambda)$ is generated by $\left\{\nu \in C: \beta^{\mathbf{m}}(\nu) \geq \eta\right\}$ via the function $g$ defined by $g(\nu)=o(\eta)$ if $\beta^{\mathbf{m}}(\nu)=\eta$ and $g(\nu)=\mathbf{c}^{\mathbf{m}}\left(\kappa, \eta, \beta^{\mathbf{m}}(\nu)\right)(\nu)$ otherwise. The ordinal $\tau^{\mathbf{m}}(\lambda)$ exists by [Mi89a, Lemma 2.6]. Now define $\beta^{C}(\nu)=\tau^{\mathbf{m}}\left(\beta^{\mathbf{m}}(\nu)\right)$. We need to show that if $f$ is any function in $K(\mathscr{F})$ then there is $\delta<\kappa$ such that for all $\nu \in C \backslash \delta$ and for all $x \in f^{\prime \prime} \nu$ we have $\nu \in x$ iff $x \in \mathscr{F}\left(\kappa, \beta^{C}(\nu)\right)$. Now this is almost immediate from the definition of $\tau^{\mathrm{m}}$, except that the statement of [Mi89a, Lemma 2.6] does not guarantee that $\delta$ is independent of $\lambda$. An examination of the proof of Lemmas 2.4 and 2.6 
of [Mi89a], however, shows that $\delta$ does in fact depend only on the function $f$ and the set $X$.

Clause (3) follows immediately from [Mi89a, Lemma 2.4] and clause (4) follows from Corollary 2.7 of [Mi89a]. In fact the proof of the covering lemma easily gives the following fact, which will be useful later in this proof and that of Lemma 2.15:

2.6. Fact. Suppose that $\nu$ is a limit point of $C$ in $C$. Then $C \cap \nu$ is a set of indiscernibles defined from $X$ in exactly the way $C$ was, and for all sufficiently large $\alpha \in C \cap \nu$

$$
\beta^{C \cap \nu}(\alpha)=\mathbf{c}^{\mathbf{m}}\left(\kappa, \beta^{\mathbf{m}}(\alpha), \beta^{\mathbf{m}}(\nu)\right)(\nu)=\mathbf{c}\left(\kappa, \beta^{C}(\alpha), \beta^{C}(\nu)\right)(\nu) .
$$

In fact the connection between $C \cap \nu$ and $X$ is stronger than that between $C$ and $X$, since in the case of $C \cap \nu$ the function corresponding to $\tau^{\mathbf{m}}$ is the identity. This fact yields the first identity in equation (1).

The proof of clause (5) will be broken into two cases, depending on whether or not $\operatorname{cf}(\kappa)=\omega$. Note that the argument for the case $\operatorname{cf}(\kappa)=\omega$ actually can be used whenever ${ }^{\mathrm{cf}(\kappa)} X \subset X$, and hence whenever $|D|^{\mathrm{cf}(\kappa)}<\kappa$. For the case of $\operatorname{cf}(\kappa)=\omega$ we have another useful fact:

2.7. Lemma. range $\left(\tau^{\mathrm{m}}\right) \supset X \cap \beta$, and if $\operatorname{cf}(\kappa)=\omega$ (or, in general, if ${ }^{\mathrm{cf}(\kappa)} X \subset$ $X)$ then $\operatorname{range}\left(\tau^{\mathbf{m}}\right)=X \cap(\beta+1)$.

Proof. First suppose that $\eta \in X \cap \beta$. Then $\mathscr{F}(\kappa, \eta)$ has a generating sequence in $V$, and hence by elementarity it has one in $X$. This generating sequence must be eventually contained in $C$, since otherwise it would have unbounded intersection with the nonstationary set $\left\{\nu: h^{\prime \prime} \nu \cap \kappa \not \subset \nu\right\}$. It follows by Corollary 2.4 that $\beta^{C}(\nu) \geq \eta$ for unboundedly many members of $C$, so there is $\lambda<\beta^{\mathbf{m}}$ such that $\tau^{\mathbf{m}}(\lambda) \geq \eta$. If $\tau^{\mathbf{m}}(\lambda)=\eta$ we are done, and if $\tau^{\mathbf{m}}(\lambda)>\eta$ then $\eta=\left[\mathbf{c}\left(\kappa, \eta, \tau^{\mathbf{m}}(\lambda)\right)\right]_{\mathscr{F}\left(\kappa, \tau^{\mathbf{m}}(\lambda)\right)}$. Now $\mathbf{c}\left(\kappa, \eta, \tau^{\mathbf{m}}(\lambda)\right)$ is in $\mathbf{m}$ since it is in $X$ and $\mathscr{P}(\kappa) \cap K(\mathscr{F}) \cap X \subset \mathbf{m}$, so $\eta=\tau^{\mathbf{m}}\left(\left[\mathbf{c}\left(\kappa, \eta, \tau^{\mathbf{m}}(\lambda)\right)\right]_{\mathscr{F}} \mathbf{m}_{(\kappa, \lambda)}\right)$.

Now suppose that $\operatorname{cf}(\kappa)=\omega$ and $\eta<\beta^{\mathrm{m}}$. Then $\mathscr{F}\left(\kappa, \tau^{\mathbf{m}}(\eta)\right)$ is generated by $\left\{\nu \in C: \beta^{\mathbf{m}}(\nu) \geq \eta\right\}$ via the function $g$ defined in Corollary 2.4. If $A$ is a countable cofinal subset of $\left\{\nu \in C: \beta^{\mathrm{m}}(\nu) \geq \eta\right\}$ then $\mathscr{F}\left(\kappa, \tau^{\mathrm{m}}(\eta)\right)$ is defined from $A$ via $g \nmid A$. Since range $(g)$ is contained in $X$ and ${ }^{\omega} X \subset X$ it follows that both $A$ and $g \mid A$ are in $X$. Thus $\mathscr{F}\left(\kappa, \tau^{\mathrm{m}}(\eta)\right)$, and hence $\tau^{\mathrm{m}}(\eta)$, are in $X$.

Now suppose that clause (5) fails and $\operatorname{cf}(\kappa)=\omega$. Then there are $\omega$ sequences $\vec{\eta}$ and $\vec{\delta}$ in $X$ such that $\vec{\delta}$ is cofinal in $\kappa$ and $s^{C^{\prime}}\left(\eta_{i}, \delta_{i}\right)<$ $s^{C}\left(\eta_{i}, \delta_{i}\right)$ for each $i$ in $\omega$. Let $\nu_{i}=s^{C}\left(\eta_{i}, \delta_{i}\right) \in X$. Then it is true in $V$, and hence by elementarity in $X$, that there is a set $A=\vec{\xi}$ of indiscernibles such that $\delta_{i}<\xi_{i}<\nu_{i}$ and $\beta^{A}\left(\xi_{i}\right) \geq \eta_{i}$. Then clause (1) implies that every sufficiently large member of $A$ is also in $C$, and Proposition 2.2 implies that $\beta^{C}\left(\xi_{i}\right)=\beta^{A}\left(\xi_{i}\right) \geq \eta_{i}$ for every sufficiently large $i$ in $\omega$. This contradicts the assumption that $\nu_{i}=s^{C}\left(\eta_{i}, \delta_{i}\right)$. 
Before considering the case of $\operatorname{cf}(\kappa)>\omega$, we note a simple corollary of the statement of Lemma 2.5:

2.8. Lemma. Any set $D$ of indiscernibles such that $|D|^{\omega}<\kappa$ is eventually contained in a set $C$ of indiscernibles satisfying the conclusion to Lemmas 2.5 and 2.15 .

Proof. Apply Lemma 2.5 with the given set $D$, let $C$ be the resulting set of indiscernibles, and let $h$ be the function given by clause (1). Then $x=\{\nu$ : $\left.h^{\prime \prime} \nu \cap \kappa \subset \nu\right\}$ is a closed, unbounded subset of $\kappa$ in $K(\mathscr{F})$ and hence is in every set $\mathscr{F}(\kappa, \eta)$. Since $D$ is a generating set it follows that every sufficiently large member $\nu$ of $D$ is in $x$ and clause (1) implies that every member of $D \cap x$ is in $C$.

Thus, if $C^{\prime}$ witnesses the failure of clause (5) then we can assume wlog that $C^{\prime} \supset C$ and there is $\delta<\kappa$ such that $C^{\prime} \backslash \delta$ is generated as in the lemma from a set $y^{\prime} \prec H_{\kappa^{++}}$. We can also assume that $\beta^{C}(\nu)=\beta^{C^{\prime}}(\nu)$ for all $\nu \in\left(C \cap C^{\prime}\right) \backslash \delta$. Now we can find $\alpha \in C \cap C^{\prime}$ so that $\alpha>\delta$ and $\alpha$ is a countable limit of ordinals $\nu_{i}=s^{C}\left(\beta^{C}\left(\nu_{i}\right), \delta_{i}\right)$ such that $s^{C^{\prime}}\left(\beta^{C}\left(\nu_{i}\right), \delta_{i}\right)<\nu_{i}$. Then $\beta^{C \cap \alpha}(\nu)=\mathbf{c}\left(\kappa, \beta^{C}(\nu), \beta^{C}(\alpha)\right)$ and $\beta^{C^{\prime} \cap \alpha}(\nu)=\mathbf{c}\left(\kappa, \beta^{C^{\prime}}(\nu), \beta^{C}(\alpha)\right)$ by Fact 2.6. Thus, $s^{C^{\prime} \cap \alpha}\left(\beta^{C \cap \alpha}\left(\nu_{i}\right), \delta_{i}\right)<s^{C \cap \alpha}\left(\beta^{C \cap \alpha}\left(\nu_{i}\right), \delta_{i}\right)$, so clause (5) fails for $C \cap \alpha$, contradicting the cofinality $\omega$ case and proving Lemma 2.5 .

2.9. Corollary. The closure of any set of indiscernibles in $\kappa$ is also a set of indiscernibles.

Proof. Apply Lemma 2.5, letting $D$ be the closure of the given set of indiscernibles. Then $D$ is eventually contained in the set of ordinals $\nu$ such that $h^{\prime \prime} \nu \subset \nu$ and hence clause (1) implies that $D$ is eventually contained in the set $C$ of indiscernibles, and hence is also a set of indiscernibles.

2.10. Corollary. Assume that $D \subset \kappa$ and $|D|^{\omega}<|\kappa|$. Then the following are equivalent:

(1) D has an assignment, and hence is a set of indiscernibles.

(2) $D$ is eventually contained in every closed unbounded subset $x$ of $\kappa$ such that $x \in K(\mathscr{F})$.

(3) $D$ is a generating sequence for some measure $\mathscr{F}(\kappa, \lambda)$.

Proof. The implication $(2) \Rightarrow(1)$ is a corollary of Lemma 2.5, taking the set $x$ to be $\left\{\nu: h^{\prime \prime} \nu \subset \nu\right\}$, where $h$ is the function of clause (1) of that lemma. It is easy to see that clause (3) implies clause (2), and clause (1) implies clause (3) with $\lambda=0$.

Note that the assumption that $D$ is small is necessary. The implications $(1) \Rightarrow(3) \Rightarrow(2)$ are valid without this assumption, but neither of the other implications hold. To see that (3) does not imply (1), assume $o(\kappa)=\kappa^{+}$and let $D$ be a Radin generic subset of $\kappa$ (see [Mi84a or Ra82]). Then $D$ generates every measure $\mathscr{F}(\kappa, \lambda)$ on $\kappa$, but we will show that there is no assignment for 
$D$. Suppose that $\beta^{D}$ were such an assignment. Then $\beta^{D \text { " }} D$ would be bounded by some $\lambda<\kappa^{+}$and there is a set $x$ such that $x \in \mathscr{F}(\kappa, \lambda)$ but $x \notin \mathscr{F}(\kappa, \eta)$ for any $\eta \neq \lambda$. Since $\beta^{D}$ is an assignment, it follows that $x \cap D$ is bounded in $\kappa$, contradicting the fact that $D$ is Radin generic and hence $x \cap D$ is unbounded. To see that (2) does not imply (3), let $\kappa$ be regular in $K(\mathscr{F})$ and generically collapse $\kappa^{+}$onto $\kappa$. Let $D$ be a diagonal intersection of the closed, unbounded subsets of $\kappa$ in $K(\mathscr{F})$. Then $D$ is closed and unbounded and satisfies (2), but since the collapse preserves stationary subsets of $\kappa$, and in particular those stationary sets which are not members of any measure $\mathscr{F}(\kappa, \lambda)$, the set $D$ does not generate any measures.

The next series of definitions leads up to Lemma 2.15, the second half of the covering lemma. The main aim is to deal with those members $\nu$ of $C$ which do not have the form $s^{C}\left(\beta^{C}(\nu), \xi\right)$ for any $\xi \in C \cap \nu$.

2.11. Definition. If $C \subset \kappa$ is a set of indiscernibles then $\alpha$ is an accumulation point in $C$ if $\alpha \in C$ and $\alpha \neq s^{C}\left(\beta^{C}(\alpha), \nu\right)$ for any $\nu \in(C \cup\{0\}) \cap \alpha$.

We would like to have a notion of an assignment $\gamma^{C}$ of the accumulation points of $C$ to ordinals $\eta \leq o(\kappa)$ which is similar to the assignment $\beta^{C}$ of indiscernibles to measures on $\kappa$. The idea should be that $\alpha$ is an accumulation point for $\lambda \leq o(\kappa)$ if $\alpha$ is a limit point of indiscernibles for arbitrarily large measures below $\mathscr{F}(\kappa, \lambda)$, but is not itself an indiscernible or limit of indiscernibles for $\mathscr{F}(\kappa, \lambda)$ or larger measures. For an individual accumulation point this description makes even less sense than the notion of an assignment of an individual indiscernible to a measure. This description does not even make sense for a single set $C$ of indiscernibles, in view of the fact that Corollary 2.9 implies that no accumulation point $\alpha$ of $C$ is a limit point of $C$. The reader should compare Definition 2.12 with Definition 1.4 of an accumulation sequence, which is (via Lemma 2.15) a special case of Definition 2.12 .

2.12. Definition. An assignment for the accumulation points of a set $C$ of indiscernibles is a function $\gamma$ such that:

(1) For all accumulation points $\alpha$ of $C$ there is an ordinal $\nu_{\alpha} \in C \cap \alpha$ such that for all sets $C^{\prime} \supseteq C$ of indiscernibles and all sufficiently large accumulation points $\alpha$ in $C$ we have $s^{C^{\prime}}\left(\gamma(\alpha), \nu_{\alpha}\right)>\alpha$.

(2) If $\gamma^{\prime}$ and $\delta$ are functions such that $\gamma^{\prime}(\alpha)<\gamma(\alpha)$ and $\delta(\alpha)<\alpha$ for all accumulation points $\alpha$ of $C$, then there is a set $C^{\prime}$ of indiscernibles such that $s^{C^{\prime}}\left(\gamma^{\prime}(\alpha), \delta(\alpha)\right)<\alpha$ for all sufficiently large accumulation points $\alpha$ of $C$.

Note that the assignment $\gamma$ for the accumulation points of $C$ is unique (except for initial segments) if it exists. We will write $\gamma^{C}$ for this assignment, and we will write $a^{C}(\lambda, \nu)$ for the least accumulation point $\alpha$ of $C$ above $\nu$ such that $\gamma^{C}(\alpha) \geq \lambda$, or $a^{C}(\lambda, \nu)=\kappa$ if there is no such accumulation point. 
Unlike the case of assignments for indiscernibles we do now know whether $\gamma^{C}$ is the restriction to $C$ of a function in $K(\mathscr{F})$.

2.13. Proposition. Suppose $C$ satisfies the conclusion of Lemma 2.5 and there is an assignment $\gamma^{C}$ for the accumulation points of $C$. Then:

(1) Except for boundedly many $\nu \in C$, if $\nu$ is a limit point of accumulation points of $C$ then $\beta^{C}(\nu) \geq \gamma^{C}(\alpha)$ for every sufficiently large $\alpha<\nu$ which is an accumulation point in $C$.

(2) For every sufficiently large ordinal $\nu \in C$ there is $\delta \in(C \cap \nu) \cup\{0\}$ such that either $\nu=s^{C}\left(\beta^{C}(\nu), \delta\right)$ or $\nu=a^{C}\left(\gamma^{C}(\nu), \delta\right)$.

Proof. Suppose that clause (1) fails for unboundedly many $\nu \in C$. For each $\nu \in C$ which is a limit point of $C$, the conclusion of Lemma 2.5 states that there is $\delta_{\nu} \in C \cap \nu$ such that $\nu=s^{C}\left(\beta^{C}(\nu), \delta_{\nu}\right)$. If the statement of the proposition fails for $\nu$ then there is an accumulation point $\alpha_{\nu}$ of $C$ such that $\delta_{\nu}<\alpha_{\nu}<\alpha$ and $\gamma^{C}\left(\alpha_{\nu}\right)>\beta^{C}(\alpha)$. Then by 2.12(2) there is a set $C^{\prime} \supset C$ of indiscernibles such that for all but boundedly many such ordinals $\nu$ we have $s^{C^{\prime}}\left(\beta^{C}(\nu), \delta_{\nu}\right)<\alpha_{\nu}<\nu=s^{C}\left(\beta^{C}(\alpha), \nu_{\alpha}\right)$, contradicting Lemma 2.5(5) and completing the proof of clause (1).

Now if $\nu \in C$ is not equal to $s^{C}\left(\beta^{C}(\nu), \delta\right)$ for any $\delta \in(C \cap \nu) \cup\{0\}$ then $\nu$ is an accumulation point in $C$. Let $\delta \in C$ be large enough that ${ }^{C}\left(\gamma^{C}(\nu), \delta\right)>\nu$. Then there can only be finitely many accumulation points $\alpha$ in $C$ in the interval between $\delta$ and $\nu$ such that $\gamma^{C}(\alpha) \geq \gamma^{C}(\nu)$, since otherwise the limit $\xi \leq \nu$ of the first $\omega$ of them would, by clause (1), have $\beta^{C}(\xi) \geq \gamma^{C}(\nu)$, contrary to the choice of $\delta$. Thus $\nu=a^{C}\left(\gamma^{C}(\nu), \alpha\right)$, where $\alpha$ is the largest of these accumulation points (or $\alpha=\delta$ if there are none).

2.14. Definition. If $X \subset o(\kappa)$ then $C$ is maximal for $X$ if

(1) there is an assignment $\gamma^{C}$ for the accumulation points of $C$,

(2) for all sets $C^{\prime}$ of indiscernibles there is $\delta<\kappa$ such that for all $\nu \in C \backslash \delta$ and all $\beta \in X \cup \beta^{C "} C \cup \gamma^{C}{ }^{C} C$ we have $s^{C}(\beta, \nu) \leq s^{C^{\prime}}(\beta, \nu)$, and

(3) if $C^{\prime} \supset C$ is a set of indiscernibles and $\gamma^{C^{\prime}}$ exists then there is $\delta<\kappa$ such that for all $\nu \in C \backslash \delta$ and all $\lambda \in X \cup \beta^{C}{ }^{C} C \cup \gamma^{C} " C$ we have $a^{C}(\lambda, \nu) \leq a^{C^{\prime}}(\lambda, \nu)$.

The set $C$ is maximal if it is maximal for the empty set.

The following lemma is the second part of the covering lemma, and is the second and last result in this paper which requires the techniques and notation of [Mi89a] for its proof. Lemmas 2.5 and 2.15 will be all that is needed for the rest of the paper.

2.15. Lemma. Suppose that $D \subset \kappa, g \in K(\mathscr{F})$, and $|D|^{\omega}<|\kappa|$. Then the set $C$ of indiscernibles of Lemma 2.5 can be chosen so that $C$ is maximal for the smallest set $Y$ containing $D$ and $C$ and closed under $g$. 
Proof. The proof of this lemma is an extension of that of Lemma 2.5. The sets $C$ and $X$ are chosen as in that proof, with the additional requirement that $g \in X$.

If $\alpha$ is an accumulation point of $C$ then $\alpha$ is not a limit point of $C$ but $C \cap \alpha$ has no largest member. In this case define $\gamma^{\mathbf{m}}(\alpha) \leq o^{\mathbf{m}}(\kappa)$ to be the least ordinal $\gamma \leq o^{\mathbf{m}}(\kappa)$ such that $\left\{\nu \in \alpha \cap C: \beta^{\mathbf{m}}(\nu) \geq \gamma\right\}$ is bounded in $C \cap \alpha$. Now recall the function $\tau^{\mathrm{m}}$ from the proof of Lemma 2.5 which takes $\beta^{\mathbf{m}}(\kappa)+1$ into $\beta+1$ and define $\gamma^{C}(\alpha)=\tau^{\mathbf{m}}\left(\gamma^{\mathbf{m}}(\alpha)\right)$.

We will first have to show that $\gamma^{C}$ satisfies Definition 2.12, so that it is an assignment for the accumulation points of $C$ and then we will have to check the other two clauses of Definition 2.14.

The definition of $\gamma^{C}$ implies that for each accumulation point $\alpha$ of $C$ there is $\nu_{\alpha} \in C \cap \alpha$ such that $s^{C}\left(\gamma^{C}(\alpha), \nu_{\alpha}\right) \geq \alpha$, and the definition of an accumulation point implies that the inequality is strict. Lemma 2.5(5) then implies that, if $C^{\prime} \supset C, s^{C^{\prime}}\left(\gamma^{C}(\alpha), \nu_{\alpha}\right) \geq s^{C}\left(\gamma^{C}(\alpha), \nu_{\alpha}\right)>\alpha$, as required for Definition 2.12(1).

To prove 2.12(2), suppose that $\gamma^{\prime}$ and $\delta$ are functions as given in the statement of clause (2) and pick $X^{\prime} \prec H_{\kappa^{++}}$as in the proof of Lemma 2.5 such that $C \cup\left\{C, \gamma^{\prime}, \delta\right\} \subset X^{\prime}$. Let $C^{\prime}$ and $h^{\prime}$ be obtained from $X^{\prime}$ as $C$ and $h$ were obtained from $X$. We claim that $C^{\prime}$ is as required. Now, as in the proof of Lemma 2.5(5), we suppose first that $\operatorname{cf}(\kappa)=\omega$ (or, more generally, that $\left.{ }^{\operatorname{cf}(\kappa)} X \subset X\right)$. If $C^{\prime}$ is not as required then there is an $\omega$ sequence $\vec{\nu}=\left(\nu_{i}: i \in \omega\right)$ cofinal in $\kappa$ such that $s^{C^{\prime}}\left(\gamma^{\prime}\left(\nu_{i}\right), \delta\left(\nu_{i}\right)\right) \geq \nu_{i}$ for all $i \in \omega$. Since range $\left(\tau^{\mathbf{m}}\right) \subset X$ by Lemma 2.7, the sequence $\left(\gamma\left(\nu_{i}\right): i \in \omega\right)$ is in $X$. By the definition of $\gamma^{C}$, the structure $X$ satisfies the statement that for all sequences $\vec{\delta}$ and $\vec{\gamma}^{\prime}$ such that $\delta_{i}<\gamma_{i}$ and $\gamma_{i}^{\prime}<\gamma\left(\nu_{i}\right)$ for all $i \in \omega$ there is a set $D$ of indiscernibles such that $s^{D}\left(\gamma_{i}^{\prime}, \delta_{i}\right)<\nu_{i}$ for all sufficiently large $i \in \omega$. By elementarity $V$, and hence $X^{\prime}$, satisfy the same statement. In particular, there is a sequence $D^{\prime} \in X^{\prime}$ such that $s^{D^{\prime}}\left(\gamma^{\prime}\left(\nu_{i}\right), \delta\left(\nu_{i}\right)\right)<\nu_{i}$. Since every sufficiently large member of $D^{\prime}$ is in $C^{\prime}$ this contradicts the choice of $\vec{\nu}$.

If $\operatorname{cf}(\kappa)>\omega$ then the same argument as that used in the proof of clause (5) of Lemma 2.5 will show that the failure of 2.12(2) at $\kappa$ implies a failure of 2.12(2) at a smaller ordinal of cofinality $\omega$, contradicting the argument above. This completes the proof that $\gamma^{C}$ is an assignment for the accumulation points of $C$. But Definition 2.14(2) is a restatement of Lemma 2.5(5), and clause 2.14(3) is proved in the same way, so completes the proof of the covering lemma.

\section{THE CLASSIFICATION OF SINGULAR CARDINALS}

Throughout this section we assume that there is no inner model of $\exists \kappa o(\kappa)=$ $\kappa^{++}$, and that $\kappa$ is a singular cardinal such that $\lambda^{\omega}<\kappa$ for all $\lambda<\kappa$. The five cases are those of Table 1 in the introduction. The definition of each case is 
given in parentheses after the case number, and the statement of the case gives the definition of the cofinal sequence, with further information in some cases. Following this statement is a proof that the statement is correct.

The set $C$ used in every case except case (1) is a set of indiscernibles given by Lemmas 2.5 and 2.15 . In some of the cases a particular set $D$ and function $g$ from Lemma 2.15 is specified.

Case 1 ( $\kappa$ is singular in $K(\mathscr{F})$ ). The least sequence in $K(\mathscr{F})$ witnessing the singularity of $\kappa$ is definable.

Case $2(\beta=\lambda+1)$. There is a maximal Prikry sequence.

Proof. Let $C$ be as in Lemmas 2.5 and 2.15, letting $D$ be a generating sequence for $\mathscr{F}(\kappa, \lambda)$. By Proposition 2.10 every sufficiently large member $\nu$ of $D$ is in $C$. Corollary 2.4 implies that $\beta^{C}(\nu)=\lambda$ for every sufficiently large ordinal $\nu \in D$ : if it were smaller for unboundedly many $\nu \in D$ then $D$ would not be a generating sequence for $\mathscr{F}(\kappa, \lambda)$, and if it were larger for unboundedly many $\nu \in D$ then $D$ would contain a generating sequence for $\mathscr{F}(\kappa, \beta)$. Thus, $C_{\lambda}=\left\{\nu \in C: \beta^{C}(\nu)=\lambda\right\}$ is cofinal in $\kappa$. On the other hand, if $\alpha$ is a limit point of $C_{\lambda}$ then $\beta^{C}(\alpha) \geq \beta$, so the set of limit points of $C_{\lambda}$ must be bounded in $\kappa$. It follows that the last $\omega$ members of $C_{\lambda}$ form a Prikry sequence, and this sequence is maximal because of the maximality of $C$.

Case $3(\operatorname{cf}(\beta)>\kappa)$. There is a maximal accumulation sequence for $\beta$ cofinal in $\kappa$.

Proof. For any $\nu<\kappa$ define $A_{\nu}=\left\{s^{C}(\lambda, \nu): \lambda<\beta\right.$ and $\left.s^{C}(\lambda, \nu)<\kappa\right\}$. We claim that $A_{\nu}$ is bounded in $\kappa$. Otherwise the set $B_{\nu}=\left\{\beta^{C}(\gamma): \gamma \in A_{\nu}\right\}$ is bounded in $\beta$ since $\left|A_{\nu}\right| \leq \kappa<\operatorname{cf} \beta$. It follows that we can find a generating sequence $D$ for some measure $\mathscr{F}(\kappa, \lambda)$ with $\lambda>\sup B_{\nu}$. By Corollary 2.10 the set $D$ is a set of indiscernibles, and by the maximality of $C$ there is an ordinal $\delta<\kappa$ such that $s^{C}(\eta, \delta) \leq s^{D}(\eta, \delta)$ for all $\eta=\beta^{C}(\alpha)$ with $\alpha \in C \backslash \delta$. We can assume that $\delta$ is large enough that $A_{\delta}$ is unbounded in $\kappa$. Now Corollary 2.4 implies that $\beta^{D}(\nu) \geq \lambda$ for all sufficiently large $\nu \in D$, so $s^{D}(\lambda, \delta)<\kappa$, and since $A_{\delta}$ is unbounded there is $\eta \in B_{\delta}$ so that $s^{C}(\eta, \delta)>s^{D}(\lambda, \delta) \geq s^{D}(\eta, \delta)$. The contradiction completes the proof that $A_{\nu}$ is bounded.

Now for any ordinal $\nu<\kappa$ define $\alpha_{\nu}$ to be $\inf \left(C \backslash \sup \left(A_{\nu}\right)\right)$, so that $\alpha_{\nu}$ is an accumulation point in $C$. We claim that $\gamma^{C}\left(\alpha_{\nu}\right)=\beta$ for every sufficiently large $\nu<\kappa$. Pick $\gamma<\beta$ so that $\gamma \geq \sup \left\{\gamma^{C}\left(\alpha_{\nu}\right): \nu<\kappa\right.$ and $\left.\gamma^{C}\left(\alpha_{\nu}\right)<\beta\right\}$, and let $D$ be a generating sequence for $\mathscr{F}(\kappa, \gamma)$. Then if $\delta_{\nu}$ is a member of $D$ above $\alpha_{\nu}$ then $s^{C}\left(\gamma^{C}\left(\alpha_{\nu}\right), \nu\right) \leq s^{D}\left(\gamma^{C}\left(\alpha_{\nu}\right), \nu\right) \leq \delta_{\nu}<\kappa$, and hence $s^{C}\left(\gamma^{C}\left(\alpha_{\nu}\right), \nu\right)<$ $\alpha_{\nu}$ by the definition of $\alpha_{\nu}$. But then $\left\{s^{C}(\gamma, \nu): \gamma \geq \gamma^{C}\left(\alpha_{\nu}\right)\right\}$ is cofinal in $A_{\nu}$, which is cofinal in $C \cap \alpha_{\nu}$, contradicting clause (1) of Definition 2.12 of an assignment for accumulation points. 
Choose $\nu_{0}$ so that $\gamma^{C}\left(\alpha_{\nu}\right)=\beta$ for all $\nu \geq \nu_{0}$, and for $i \geq 0$ define $\nu_{i+1}=$ $\alpha_{\nu_{i}}$. Then Proposition 2.13 implies that $\kappa=\sup _{i} \nu_{i}$. Thus $\left(\nu_{i}: i \in \omega\right)$ is an accumulation sequence for $\beta$, and it is maximal by Definition 2.14(3).

Moti Gitik has constructed a model in which Case 3 holds. This construction, which uses techniques of [Gi86] and is considerably more difficult then that used to construct a model for the other cases, is given (for a slightly different situation) in [Gi89].

Case $4\left(\mathrm{cf}^{K(\mathscr{F})}(\beta)=\kappa\right)$. There is a function $g \in K(\mathscr{F})$, a maximal set $C$ of indiscernibles, and an ordinal $\delta<\kappa$ such that if $\delta<c_{0}<\kappa$ then the sequence defined by $c_{i+1}=s^{C}\left(g\left(c_{i}\right), c_{i}\right)$ is cofinal in $\kappa$.

The cofinal sequence $\mathbf{c}$ need not be definable, but if $\mathscr{A}$ is the set of all cofinal sequences d satisfying $d_{i+1}=s^{C^{\prime}}\left(g^{\prime}\left(d_{i}\right), d_{i}\right)$ for some continuous function $g^{\prime} \in K(\mathscr{F})$ taking $\kappa$ cofinally into $\beta$, some set $D$ of indiscernibles maximal for $g^{\prime \prime} D$, and some choice of $d_{0}$, then (1) if $\mathbf{d}$ is a sequence in $\mathscr{A}$ which has unbounded intersection with $C$ then there is $j<\omega$ such that $d_{j} \in C$ and $d_{i+1}=s^{C}\left(g\left(d_{i}\right), d_{i}\right) \in C$ for each $i \geq j$, and (2) if $\mathbf{c}$ and $\mathbf{d}$ are two sequences in $\mathscr{A}$ then either $\mathbf{c}$ and $\mathbf{d}$ are eventually equal or there are integers $n$ and $m$ such that $c_{n+i}<d_{m+i}<c_{n+i+1}$ for all $i \in \omega$.

This definability statement can be strengthened slightly: there is a definable set $\mathscr{K}$ of nondecreasing functions $k$ such that $(1)$ range $(k) \subset$ domain $(k) \subset \kappa$ for all $k \in \mathscr{K},(2)$ for any set $Y \subset \kappa$ with $|Y|^{\omega}<\kappa$ there is $k \in \mathscr{K}$ with $Y \subset$ domain $k,(3)$ any two members of $\mathscr{K}$ agree on their common domain (except on an initial segment), and (4) for any $k \in \mathscr{K}$ there is $\nu<\kappa$ such that $\left\{k^{n}(\nu): n \in \omega\right\}$ is cofinal in $\kappa$.

Proof. Let $C$ be as in Lemmas 2.5 and 2.15, with $g$ being the least continuous function in $K(\mathscr{F})$ taking $\kappa$ cofinally into $\beta$. Note that if $h$ is as given by Proposition 2.2 then the set of $\nu<\kappa$ such that $g(\nu)=\sup \left(h^{\prime \prime}(\nu) \cap \beta\right)$ is a closed and unbounded subset of $\kappa$ which is in $K(\mathscr{F})$ and hence is eventually contained in $C$. Choose $\delta<\kappa$ so that $\nu \in C \backslash \delta$ implies that $\beta^{C}(\nu)<\beta$ and $g(\nu)=\sup \left(h^{\prime \prime} \nu \cap \beta\right)$. We claim that if $\mathbf{c}$ is any sequence such that $\delta \leq c_{0} \in C$ and $c_{i+1}=s^{C}\left(g\left(c_{i}\right), c_{i}\right)$ for all $i \in \omega$ then $\mathbf{c}$ is cofinal in $\kappa$. Suppose to the contrary that $\nu=\operatorname{supc}<\kappa$. Then $\nu$ is in $C$, since it is a limit point of $C$ of cofinality $\omega$. Then since $\beta^{C}(\nu) \in h^{\text {" }} \nu$ we have $\beta^{C}(\nu) \in h^{\prime \prime} c_{i}$ for all sufficiently large $i<\omega$. But then $g\left(c_{i}\right) \geq \beta^{C}(\nu)$, and since Lemma 2.5(4) implies that $\nu=s^{C}\left(\beta^{C}(\nu), c_{i}\right)$ for all sufficiently large $i \in \omega$, this contradicts the definition of $c_{i+1}<\nu$.

Now suppose that $\mathbf{d} \in \mathscr{A}$, so that $d_{i+1}=s^{D}\left(g^{\prime}\left(d_{i}\right), d_{i}\right)$ for some continuous function $g^{\prime}$ and some set $D$ of indiscernibles which is maximal for $g^{\prime \prime \prime} D$. Then the set of ordinals $\nu$ such that $g^{\prime}(\nu)=g(\nu)$ is closed and unbounded and hence eventually contains $C \cup D$. Pick $\delta<\kappa$ so that $g(\nu)=g^{\prime}(\nu)$ and $s^{C}(g(\nu), \nu)=s^{C^{\prime}}(g(\nu), \nu)$ for all $\nu \in(C \cap D) \backslash \delta$. Now suppose that $d_{j} \in C \backslash \delta$. Then for all $i \geq j$ we have $d_{i+1}=s^{D}\left(g^{\prime}\left(d_{i}\right), d_{i}\right)=s^{C}\left(g\left(d_{i}\right), d_{i}\right) \in C$. Finally, 
if $\mathbf{c}$ and $\mathbf{d}$ are any two members of $\mathscr{A}$ then by the first part of this paragraph we can assume that $\mathbf{c}$ and $\mathbf{d}$ are both defined from the same maximal set $C$ and function $g$. Then, if we set $k(\nu)=s^{C}(g(\nu), \nu), k: \kappa \rightarrow \kappa$ is nondecreasing, and $c_{i+1}=k\left(c_{i}\right)$ and $d_{i+1}=k\left(d_{i}\right)$ for each $i<\omega$. It follows that $\mathbf{c}$ and d are either eventually equal or eventually alternating, as required by the last sentence of the second paragraph.

Finally, let $\mathscr{K}$ be the set of functions $k$ such that for some continuous cofinal function $g: \kappa \rightarrow \beta$, some set $Y$, and some set $C$ of indiscernibles which is maximal for $Y$, where $C \subset Y$ and $Y$ is closed under $g$, the function $k$ with domain $Y \cap \kappa$ is defined by $k(\nu)=s^{C}(g(\nu), \nu)$. Then $\mathscr{K}$ satisfies the last paragraph.

The simplest examples of models for Case 4 are given under the assumption that $o(\kappa)=\kappa$, either by adding a Radin generic set $C$ or by a simple modified Prikry forcing to add only a sequence $\mathbf{c}=\left(c_{i}: i \in \omega\right)$ of indiscernibles such that $o\left(c_{i+1}\right)=c_{i}$. Both of these examples have witnesses which are outright definable. If $C \subset \kappa$ is Radin generic over $K(\mathscr{F})$ then the set of limit points of $C$ is equal to the set of ordinals $\nu<\kappa$ such that there is a set of indiscernibles cofinal in $\nu$ and hence is definable, but this is Radin generic for the sequence of all measures on $\kappa$ except the order 0 measure. In the other case, the sequence c added by the modified Prikry forcing is definable, since it is the only member of $\mathscr{A}$ such that $o\left(c_{0}\right)=0$ and $o\left(c_{i+1}\right)=c_{i}$ for all $i \in \omega$ (and $\mathscr{A}$ is the set of sequences which are eventually equal to c). The techniques of [Mi84a] or of [Gi86], however, can be used to define a model in which no witness is definable even up to initial segments.

Case $5\left(\mathrm{cf}^{K(\mathscr{F})}(\beta)<\kappa\right)$. Let $Y \in K(\mathscr{F})$ be the least subset of $\beta$ in the order of construction of $K(\mathscr{F})$ such that $Y$ is cofinal in $\beta$ and $|Y|^{K(\mathscr{F})}=\operatorname{cf}^{K(\mathscr{F})}(\beta)$. Then there is a set $C$ of indiscernibles which is maximal for $Y$, and for any such set $C$ there is $\nu<\kappa$ such that the set $\left\{s^{C}(\lambda, \nu): \lambda \in Y\right\}$ is cofinal in $\kappa$. Furthermore this sequence is, up to initial segments, independent of the choice of $C$ and $\nu$.

Proof. To get the set $C$ of indiscernibles which is maximal for $Y$, let $D=$ $\operatorname{cf}^{K(\mathscr{F})}(\beta)$, let $g$ be a function in $K(\mathscr{F})$ taking $D$ onto $Y$, and apply Lemma 2.15 .

From the choice of $Y, \lim \sup \left\{\beta^{C}(\nu): \nu \in C\right\}=\beta$. For $\nu<\kappa$ set $A_{\nu}=\left\{s^{C}(\lambda, \nu): \lambda \in Y\right\}$ and suppose that $\gamma_{\nu}=\sup A_{\nu}<\kappa$. Then $\gamma_{\nu}$ is in the closure $\bar{C}$ of $C$, and $\beta^{\bar{C}}\left(\gamma_{\nu}\right) \geq \sup (Y)=\beta$ for sufficiently large $\nu<\kappa$. It follows that there is some $\nu<\kappa$ such that $A_{\nu}$ is unbounded in $\kappa$. The maximality of $C$ implies that $A_{\nu}$ is unique except for an initial segment.

\section{THE RAMSEY THEOREM}

Now we can apply the results of $\S 3$ to the proof of Theorem 1.2. The strategy is to define a function $f:[\mathrm{ORD}]^{\omega} \rightarrow \Re_{f}$ such that for no $\nu \in$ ORD is there any 
$\mathbf{c} \in[\nu]^{\omega}$ such that $f$ is homogeneous on $\mathbf{c}$. We will define $f(\mathbf{c})$ by recursion on $\sup (\mathbf{c})$. The induction will preserve the property that $f(\mathbf{c})=f(\mathbf{d})$ whenever c and $\mathbf{d}$ are equal except for an initial segment. In Case 5 this independence of initial segment will be needed for the induction hypothesis.

We are given $f_{0}:\left[\left(2^{\omega}\right)^{+}\right]^{\omega} \rightarrow \Re_{f}$ so that for no $\nu<\left(2^{\omega}\right)^{+}$is there any sequence $\mathbf{c} \in[\nu]^{\omega}$ which is homogeneous for $f_{0}$. For the final sentence of Theorem 1.2, in which $f_{0}(\mathbf{c})$ is assumed to be independent of initial segments of $\mathbf{c}$, we will set $f\left[\left[\left(2^{\omega}\right)^{+}\right]^{\omega}\right.$ equal to $f_{0}$. For the general case we will still set $f(\mathbf{c})=f_{0}(\mathbf{c})$ whenever $\nu=\sup (c)<\left(2^{\omega}\right)^{+}$and $\operatorname{cf}^{K(\mathscr{F})}(\nu)$ is measurable in $K(\mathscr{F})$, but if $\nu<\left(2^{\omega}\right)^{+}$and $\mathrm{cf}^{K(\mathscr{F})}(\nu)$ is not measurable in $K(\mathscr{F})$ then we will use a modification of an argument of Spector's [Sp] to explicitly construct $f(\mathbf{c})$ so that it is independent of initial segments.

Fix $\nu<\left(2^{\omega}\right)^{+}$such that $\mathrm{cf}^{K(\mathscr{F})}(\nu)$ is not measurable in $K(\mathscr{F})$. There is no ultrafilter $U$ on $\mathscr{P}(\nu) \cap K(\mathscr{F})$ such that $\operatorname{ult}(K(\mathscr{F}), U)$ is well founded, since otherwise if $f$ is a nonconstant function in $K(\mathscr{F})$ such that $[f]_{U}$ is minimal, then $U^{\prime}=\left\{x: f^{-1}(x) \in U\right\}$ is a normal ultrafilter $U^{\prime}$ on $\operatorname{cf}^{K(\mathscr{F})}(\nu)$ such that $\operatorname{ult}\left(K(\mathscr{F}), U^{\prime}\right)$ is well founded. This contradicts the assumption that $\mathrm{cf}^{K(\mathscr{F})}(\nu)$ is not measurable in $K(\mathscr{F})$, since [Mi?, Lemma 7.16] implies that any such ultrafilter would be on the sequence $\mathscr{F}$.

Now let $\mathbf{c}$ be a cofinal member of $[\nu]^{\omega}$ and let $U_{\mathbf{c}}$ be $\{x \subset \nu: \mathbf{c} \backslash x$ is bounded\}, the filter generated by $\mathbf{c}$. We consider first the case in which $U_{c}$ is not an ultrafilter on $K(\mathscr{F})$. In this case let $x_{\mathrm{c}} \subset \nu$ be the least set $x$ in the order of construction of $K(\mathscr{F})$ such that neither $x$ nor $\nu \backslash x$ are in $U_{c}$, define

$$
g(\mathbf{c})=\left\{n \in \omega: \exists i c_{i} \in x_{\mathbf{c}} \text { and } c_{i+n} \in x_{\mathbf{c}} \text { and } \forall j\left(0<j<n \Rightarrow c_{i+j} \notin x_{\mathbf{c}}\right)\right\} \text {, }
$$

and let $f(\mathbf{c})=[g(\mathbf{c})]_{f} \in \Re_{f}$. Then $f(\mathbf{c})$ is independent of initial segments of c. Now suppose $y \in[g(\mathbf{c})]^{\omega}$. We will show that there is $\mathbf{d} \in[\mathbf{c}]^{\omega}$ such that $g(\mathbf{d})=y$, so that $f$ is not homogeneous on c. If $y=\left\{y_{i}: i \in \omega\right\}$ then the members of $\mathbf{d}$ are chosen in increasing order by picking, in the $i$ th stage, one member of $\mathbf{c} \cap x_{\mathbf{c}}$ followed by $y_{i}$ members of $\mathbf{c} \backslash x_{\mathbf{c}}$. This is always possible since neither $x_{c}$ nor its complement are in $U_{c}$, and this procedure ensures that neither $x_{\mathrm{c}}$ nor its complement is in $U_{\mathbf{d}}$. Thus $U_{\mathrm{d}}$ is not an ultrafilter, and $x_{\mathbf{d}}=x_{\mathbf{c}}$ since $U_{\mathbf{d}} \supset U_{\mathbf{c}}$. It follows that $g(\mathbf{d})=y$, as desired.

Now consider the case that $U_{c}$ is an ultrafilter but $K(\mathscr{F})^{\kappa} / U_{\mathrm{c}}$ is not well founded. In this case define $\left(h_{n}: n \in \omega\right)$ by recursion on $n: h_{0}$ is the least function in the order of construction of $K(\mathscr{F})$ such that $K(\mathscr{F})^{\kappa} / U_{\mathrm{c}}$ is not well founded below $\left[h_{0}\right]_{U_{\mathrm{c}}}$ and $h_{n+1}$ is the least function in the order of construction of $K(\mathscr{F})$ such that $\left[h_{n+1}\right]_{U_{\mathrm{c}}}<\left[h_{n}\right]_{U_{\mathrm{c}}}$ and $K(\mathscr{F})^{\kappa} / U_{\mathrm{c}}$ is not well founded below $\left[h_{n+1}\right]_{U_{\mathrm{c}}}$. Now for $i \in \omega$ define $n_{i}$ to be the least $n$ such that $h_{n+1}\left(c_{i}\right) \nless h_{n}\left(c_{i}\right)$, define $g(\mathbf{c})=\left\{n_{i}: i \in \omega\right\}$, and set $f(\mathbf{c})=[g(\mathbf{c})]_{f} \in \Re_{f}$. Then $f$ is independent of initial segments of $\mathbf{c}$. Let $\mathbf{d}$ be any member of $[\mathbf{c}]^{\omega}$. Then $U_{\mathrm{d}}=U_{\mathrm{c}}$ and hence the sequence $\left(h_{n}: n \in \omega\right)$ is unchanged. Thus for 
any infinite subset $y$ of $g(\mathbf{c})$ we can set $\mathbf{d}=\left\{c_{i} \in \mathbf{c}: n_{i} \in y\right\}$ so that $y=g(\mathbf{d})$ and hence $\mathbf{c}$ is not homogeneous for $f$.

Now we define $f(\mathbf{c})$ for $\sup (\mathbf{c})>\left(2^{\omega}\right)^{+}$by recursion on $\kappa=\sup \mathbf{c}$, ensuring that if $\sup (\mathbf{c})>\left(2^{\omega}\right)^{+}$then $f(\mathbf{c})$ does not depend on initial segments of $\mathbf{c}$. Since $\operatorname{cf}(\kappa)=\omega<\kappa$, we can use the five cases from the last section:

Case 1 ( $\kappa$ is singular in $K(\mathscr{F})$ ). Let $h \in K(\mathscr{F})$ be the least continuous, cofinal function in the order of construction of $K(\mathscr{F})$ which takes $\mathrm{cf}^{K(\mathscr{F})}(\kappa)$ cofinally into $\kappa$, and let $f(\mathbf{c})=f\left(\mathbf{c}^{h}\right)$, where $c^{h}(i)$ is the ordinal $\nu<\mathrm{cf}^{K(\mathscr{F})}(\kappa)$ such that $h(\nu) \leq c_{i}<h(\nu+1)$.

Cases 2 and $3\left(\beta=\gamma+1\right.$ or $\left.\operatorname{cf}^{K(\mathscr{F})}(\beta)>\kappa\right)$. In these cases $\kappa$ has a cofinal $\omega$ sequence $C$ which is either a maximal Prikry sequence or a maximal sequence of accumulation points. In either case $C$ is definable up to initial segments. Define $g_{C}(\mathbf{c})=\left\{\left|\left(c_{i+1} \backslash c_{i}\right) \cap C\right|: i \in \omega\right\}$ and set $f(\mathbf{c})=\left[g_{C}(\mathbf{c})\right]_{f}$. Then $f$ does not depend on the maximal sequence $C$ and is independent of initial segments of $\mathbf{c}$. If $\mathbf{c}$ is any sequence in $[\kappa]^{\omega}$ then for any infinite $y \subset \omega$ there is $\mathbf{c}^{\prime} \in[\mathbf{c}]^{\omega}$ such that $y \backslash g\left(\mathbf{c}^{\prime}\right)$ is infinite, and thus c cannot be homogeneous for $f$.

Case $4\left(\operatorname{cf}^{K(\mathscr{F})}(\beta)=\kappa\right)$. Let $\mathscr{K}$ be the class of functions given by the last paragraph of Case 4 of $\S 3$. If $\kappa=\operatorname{supc}$ and $k$ is a member of $\mathscr{K}$ such that c $\subset$ domain $(k)$ then define $g_{k}(\mathbf{c})=\left\{n \in \omega: \exists i \in \omega k^{n-1}\left(c_{i}\right)<c_{i+1} \leq k^{n}\left(c_{i}\right)\right\}$. If $k^{\prime}$ is any other member of $\mathscr{K}$ with domain $\left(k^{\prime}\right) \supset \mathbf{c}$ then there is $i \in \omega$ such that $k^{\prime}$ and $k$ agree on their common domain above $c_{i}$. It follows that $g_{k}(\mathbf{c})=g_{k^{\prime}}(\mathbf{c})$ except on an initial segment, so that $f(\mathbf{c})=\left[g_{k}(\mathbf{c})\right]_{f}$ does not depend on $k$ and is independent of initial segments of c. If $y$ is any infinite subset of $\omega$ then there is $\mathbf{d} \in[\mathbf{c}]^{\omega}$ such that $y \backslash g_{k}(\mathbf{d})$ is infinite, and hence c is not homogeneous for $f$.

Case $5\left(\lambda=\operatorname{cf}^{K(\mathscr{F})}(\beta)<\kappa\right)$. The analysis from the last section shows that in this case there is a function $\sigma: \lambda \rightarrow \kappa$ which is continuous, cofinal, and definable up to initial segments. If $\sup (\mathbf{c})=\kappa$ then set $f(\mathbf{c})=f\left(\mathbf{c}^{\sigma}\right)$, where the sequence $\mathrm{c}^{\sigma}$ is defined as in Case 1: $c_{i}^{\sigma}$ is the ordinal $\xi<\lambda$ such that $\sigma(\xi) \leq c_{i}<\sigma(\xi+1)$. Then there is no homogeneous sequence $\mathbf{c}$ for $f$ in $[\kappa]^{\omega}$.

Now suppose that $f\left(\mathbf{c}^{\sigma}\right)$ is independent of initial segments of $\mathbf{c}^{\sigma}$. Since the function $\sigma$ is definable up to initial segments, it follows that $f(\mathbf{c})$ is independent of initial segments of $\mathbf{c}$, and that $f$ is independent of $\sigma$ and hence is definable.

Now if $\lambda>\left(2^{\omega}\right)^{+}$then $f\left(\mathbf{c}^{\sigma}\right)$ is independent of initial segments of $\mathbf{c}^{\sigma}$ by the induction hypothesis. If, on the other hand, $\lambda<\left(2^{\omega}\right)^{+}$then $f\left(\mathbf{c}^{\sigma}\right)$ is still independent of initial segments of $\mathbf{c}^{\sigma}$, either because $f \backslash\left[\left(2^{\omega}\right)^{+}\right]^{\omega}=f_{0}$ is independent of initial segments, or because $\lambda \leq o(\kappa)$ implies that $\lambda$ is smaller than the first measurable cardinal in $K(\mathscr{F})$, so that $\mathrm{cf}^{K(\mathscr{F})}(\lambda)$ is not measurable in $K(\mathscr{F})$ and $f\left(\mathbf{c}^{\sigma}\right)$ was explicitly constructed to be independent of initial segments. 


\section{THE FULL COVERING LEMMA AND OTHER OPEN PROBLEMS}

The obvious open questions concerning the results of this paper deal with possible strengthenings of the covering lemma, Lemmas 2.5 and 2.15. The problem of constructing a core model for larger cardinals is beyond the scope of this paper, but a solution to the following question might give some information about the existence and properties of such models.

5.1. Question. Is there, under any large cardinal hypothesis, a class model $M$ of ZFC which contains a singular cardinal $\kappa$ such that there is no witness in $M$ to the singularity of $\kappa$ which is, in some reasonable sense, definable.

The criterion for definability of the witness has deliberately been left vague. The weakest positive answer to the question would be a model violating the naive generalization of the definability from $\S 4$, that is, a model in which there is a singular strong limit cardinal $\kappa$ such that either $\operatorname{cf}(\kappa)>\omega$ and no witness is definable up to initial segments, or $\operatorname{cf}(\kappa)=\omega$ and there is no class $\mathscr{K}$ witnessing the cofinality as in Case 4 of $\S 4$. A stronger example would be a model in which the hypothesis to Theorem 1.2 holds, that is, $\kappa \rightarrow(\omega)_{\Re_{f}}^{\omega}$ holds for some cardinal $\kappa$ above $\left(2^{\omega}\right)^{+}$but for no cardinal $\kappa$ below $\left(2^{\omega}\right)^{+}$. For the strongest positive answer one might ask for a forcing which makes $\kappa$ singular and has homogeneity analogous to the forcing for adding Cohen subsets of $\kappa$.

We can consider two general questions concerning the covering lemma for sequences of measures:

5.2. Question. What is the full covering lemma: the strongest (and most elegant) statement of the covering lemma for sequences of measurable cardinals.

5.3. Question. Can the full covering lemma from Question 5.1 be stated, as in this paper, without the use of mice?

The first question is presumably inconsistent in asking for both strength and elegance. Lemmas 2.5 and 2.15 could, for example, be strengthened ad nauseum by adding more clauses. One can, however, hope for a simple basic statement to which extra information can be added by clauses which are natural, reasonably simple, and independent of each other. Conjecture 5.4 below would, if true, give a start on such an approach. In order to state it we have to first observe that the definition of a set of indiscernibles can be extended to allow for a set $C$ of indiscernibles which contains indiscernibles for measures on more that one cardinal-that is, to a system of indiscernibles as defined in [Mi84a]. This is done by defining an assignment for a set $C$ of indiscernibles to consist of two functions, $\alpha^{C}$ and $\beta^{C}$, so that an ordinal $\nu \in C$ is an indiscernible for the measure $\mathscr{F}\left(\alpha^{C}(\nu), \beta^{C}(\nu)\right)$. Then the functions $s^{C}$ and $a^{C}$ now have three variables: $s^{C}(\alpha, \beta, \xi)$ is the least $\nu \in C \backslash \xi+1$ such that $\alpha^{C}(\nu)=\alpha$ and $\beta^{C}(\nu)=\beta$, and similarly for $a^{C}(\alpha, \gamma, \xi)$.

5.4. Conjecture. Suppose that $x \subset \kappa$ and $|x| \cup \omega_{1}<|\kappa|$. Then there is a set $C$ of indiscernibles, a function $h \in K(\mathscr{F})$, and an ordinal $\delta<\kappa$ such that 
$x \subset y$, where $y$ is the smallest set such that $\delta \subset y, C$ is maximal for $y$, and $y$ contains every ordinal $\nu$ such that any of the following conditions hold:

(i) $\nu \in h^{\prime \prime} y \cap \nu$.

(ii) $\nu=s^{C}(\alpha, \beta, \xi)$, where $\alpha$ and $\beta$ are in $h^{\prime \prime}(\nu \cap y)$ and $\xi$ is in $\nu \cap y$.

(iii) $\nu=a^{C}(\alpha, \gamma, \xi)$, where $\alpha$ and $\gamma$ are in $h^{\text {“c }}(\nu \cap y)$ and $\xi$ is in $\nu \cap y$.

The difficulty here is in getting $\beta^{C}(\nu)$ and $\gamma^{C}(\nu)$ to be members of $h^{\text {“ }}(\nu \cap y)$. Proposition 2.2 says only that $\beta^{C}(\nu) \in h^{\prime \prime} \nu$, and we do not even have this much for $\gamma^{C}$. The advantage of using the formulation of the covering lemma which uses mice is that both $\beta^{\mathbf{m}}(\nu)$ and $\gamma^{\mathbf{m}}(\nu)$ are in $h^{\mathbf{m} \text { “ }}(\nu \cap y)$. A positive answer to Conjecture 5.4 would follow from a positive answer to the following question:

5.5. Question. Is the function $\tau^{\mathrm{m}}$ defined in the proof of Lemma 2.5 a member of $K(\mathscr{F})$ ?

If the answer to this question is "no" then there must be a mouse with a repeat point.

Note that one strengthening of Conjecture 5.4 follows immediately from its statement: a recursion on $\kappa$ can be used to replace $\delta<\kappa$ with the stronger condition $|\delta|=|x| \cup \omega_{1}$.

Another way of approaching Question 5.2 is to ask whether the covering lemma for sequences of measures can be made to look more like the DoddJensen covering lemma for a single measure, which is both more elegant and (given the hypothesis that $0^{\dagger}$ does not exist) stronger than any statement of the covering lemma for sequences of measures. The next two questions address this problem.

5.6. Question. Suppose that $\kappa$ is a singular cardinal in $V$ and is regular in $K(\mathscr{F})$. Must $\kappa$ have been made singular by a forcing extension? That is, is it always true that there is a model $M$ and a set generic extension $M[G]$ of $M$ such that $\kappa$ is regular in $M$ and singular in $M[G]$.

Note that the answer is almost certainly no if $M$ is required to be equal to $K(\mathscr{F})$. In [Fr], S. Friedman starts with a model $L(\mu)$ and uses Jensen coding theory [BJW] to construct a class generic extension $M$ such that the measure $\mu$ extends to a measure in $M$ and there is a real number $a$ in $M$ which is not set generic over $L(\mu)$. The same construction is probably possible starting with a model $L(\mathscr{F})$ such that $o(\kappa)=\omega$, so that each of the measures $\mathscr{F}(\kappa, n)$ for $n<\omega$ extends to a measure in $M$. In this case it is possible to make $\kappa$ singular by modifying Prikry forcing to add a countable set $C$ of indiscernibles such that $\left\{\beta^{C}(\nu): \nu \in C\right\}$ is equal to the set of integers which code up some initial segment of $a$. Then the real $a$ would be coded by any witness to the singularity of $\kappa$ in $M(C)$, and hence $M(C)$ cannot be set generic over $L(\mathscr{F})$.

The final question is not a new problem, but a reminder of work which has been neglected in all of the author's research starting with [Mi84]: 
5.7. Question. Can $2^{\omega}$ and $\lambda^{\omega}$ be replaced with $\omega_{1}$ and $\lambda \cup \omega_{1}$ throughout this paper?

The analogy with the Dodd-Jensen covering lemma suggests it can. The assumption that ${ }^{\omega} X \subset X$ has been used rather heavily in [Mi84b, Mi?, and Mi89a] as well as in this paper, so an answer to this paper would require a good deal of work even if the mathematics turns out to be routine. It shold be noted that our willingness to assume that $\lambda^{\omega}<\kappa$, but not that $\lambda^{\mathrm{cf}(\kappa)}<\kappa$, is somewhat artificial. If we made this second assumption then (as noted in the proofs of Lemmas 2.5 and 2.15) several of the arguments could be simplified since the proof for $\operatorname{cf}(\kappa)=\omega$ would apply to the case of uncountable cofinality as well.

\section{REFERENCES}

[D] A. Dodd, The core model, London Math. Soc. Lecture Notes, no. 61, Cambridge Univ. Press, Cambridge, 1982.

[Fr] S. Friedman, Coding over a measurable cardinal, preprint.

[Gi86] M. Gitik, Changing cofinalities and the nonstationary ideal, Israel J. Math. 56 (1986), 280-314.

[Gi89] _ On the strength of $\neg S C H$, handwritten notes.

[BJW] A. Beller, R. Jensen, and P. Welch, Coding the universe, London Math. Soc. Lecture Notes, no. 47, Cambridge Univ. Press, Cambridge, 1982.

[Ma] M. Magidor, Changing cofinality of cardinals, Fund. Math. 99 (1978), 61-71.

[Mi74] W. J. Mitchell, Sets constructible from sequences of ultrafiters, J. Symbolic Logic 39 (1974), 57-66.

[Mi84a] _ Indiscernibles, skies and ultrafilters, Contemp. Math. 31 (1984), 161-182.

[Mi84b] _ The core model for sequences of measures. I, Math. Proc. Cambridge Philos. Soc. 95 (1984), 41-58.

[Mi?] _ The core model for sequences of measures. II, submitted to Math. Proc. Cambridge Philos. Soc. (to appear).

[Mi89a] _ On the singular cardinal hypothesis, Trans. Amer. Math. Soc. (submitted).

[Mi89b] __, An infinitary Ramsey property, Ann. Pure Appl. Logic (submitted).

[P] K. Prikry, Changing measurable into accessible cardinals, Dissertationes Math. (Rozprawy Mat.) 68 (1971), 359-378.

[Ra82] L. B. Radin, Adding closed cofinal sequences to large cardinals, Ann. Math. Logic 22 (1982), 243-261.

[Sp] M. Spector, Natural sequences of mathematics which are independent of $V=L, v=L[\mu]$, etc., preprint.

Department of Mathematics, University of Florida, Gainesville, Florida 32611

E-mail address: mitchell@math.ufl.edu 